\title{
HYDROGEN EMBRITTLEMENT OF TRIP STEEL AFTER PREVIOUS DEFORMATION
}

\author{
Petra VÁŇOVÁ, Jaroslav SOJKA, Mária MELICHEROVÁ, Kateřina KONEČNÁ, \\ Tat'ána RADKOVSKÁ ${ }^{1}$
}

1VSB - Technical University of Ostrava, Ostrava, Czech Republic, EU, petra.vanova@vsb.cz

https://doi.org/10.37904/metal.2020.3502

\begin{abstract}
The aim of the work was to obtain information about the effect of hydrogen in pre-deformed samples made of TRIP 780 steel and during deformation, when retained austenite transforms into martensite. Electrolytic hydrogenation took place both under the simultaneous action of deformation and before the tensile test itself. After hydrogenation, TRIP steel showed an increase in the yield strength and a decrease in the tensile strength. Elongation at fracture also decreased significantly by more than $20 \%$ compared to samples that were only pre-deformed. The occurrence of deformation-induced martensite in the microstructure of TRIP steels significantly contribute to the susceptibility of these steels to hydrogen embrittlement. For samples with $5 \%$ predeformation, the value of the hydrogen embrittlement index was found to be $78.1 \%$, for samples with $10 \%$ predeformation it further increased to $86.2 \%$. From these results, hydrogen led to a degradation of mechanical properties with increasing percentage of deformation and the same time the obtained results demonstrate a significant susceptibility of TRIP steels to hydrogen embrittlement. The article also contains results of a fractographic analysis, which also showed the occurrence of typical signs of hydrogen embrittlement, which are a quasi-cleavage fracture and fisheyes.
\end{abstract}

Keywords: Hydrogen embrittlement, TRIP steel, deformation, fractography

\section{INTRODUCTION}

TRIP (TRansformation Induced Plasticity) steels, i.e. steels with transformation-induced plasticity, belong among the multiphase steels. These are modern high-strength steels of the AHSS (Advanced High Strength Steels) type. TRIP steels are among the materials that are widely used in the automotive industry, especially for their mechanical properties, which require high strength values in the range of 500-1000 MPa, high ductility of $20-40 \%$ and the ability to absorb large amounts of dynamic energy under high strain rates so that increasingly stringent body safety requirements are met. Other criteria that these steels must meet due to their use in the automotive industry on body parts are their good weldability and formability, as well as their suitability for galvanizing [1,2].

Hydrogen embrittlement is a very serious problem causing the degradation of structural components, especially in steels with a predominant body centered cubic lattice. All components made of steel that are chemically or electrochemically surface treated can absorb hydrogen that is evolved during these processes. Absorbed hydrogen can cause premature failure, especially for components made of high strength steels. The heat treatment of the components that should follow the surface treatment minimizes the risk of damage, but this process can never be considered as $100 \%$ efficient. Hydrogen embrittled components usually fail when a load is applied below the level of normal fracture stress, and most hydrogen embrittlement failures occur relatively shortly after assembly within a few minutes to weeks $[3,4]$.

The study of hydrogen embrittlement in TRIP steels is specific due to the presence of several phases in the microstructure of steel, which differ in their crystallographic arrangement, and thus in different diffusion characteristics and solubility of hydrogen in austenite and ferrite, ferritic bainite and martensite. In addition, 
during the deformation-induced phase transformation of retained austenite to martensite these parameters change [1-3].

One of the main goals of this work is to study the susceptibility of TRIP steels to hydrogen embrittlement. The susceptibility to hydrogen embrittlement of TRIP steels will be evaluated mainly by means of tensile tests at a slow rate of deformation, the so-called Slow Strain Rate Test (SSRT), on pre-hydrogenated samples or samples electrolytically hydrogenated during the tensile test. The changes in mechanical properties caused by hydrogen embrittlement will then be expressed by the hydrogen embrittlement index expressing the relative change in the elongation at fracture due to the impact of hydrogen. In addition to the decrease in the elongation at fracture and in some cases the strength properties, the study will also focus on the identification of fracture initiation sites and the characterization of changes in the micromechanism of failure.

\section{EXPERIMENTAL PART}

\subsection{Material description}

TRIP $780 \mathrm{C}-\mathrm{Mn}-\mathrm{Si}$ steel was used for this experiment. This material was supplied after one-stage hot rolling and after two-stage cold rolling. The strength and plastic characteristics of this steel are as follows: the tensile strength is about $780-900 \mathrm{MPa}$, the yield strength is about $450-550 \mathrm{MPa}$ and the elongation at fracture of this steel is greater than $23 \%$. This steel is mainly used in the automotive industry, due to its excellent properties and excellent energy absorption capacity. It is used to produce bumpers, safety reinforcements and the like. The chemical composition of the studied TRIP 780 steel is given in Table 1.

Table 1 Chemical composition of the TRIP 780 steel (wt\%) determined by the GDOES method

\begin{tabular}{|c|c|c|c|c|c|c|c|}
\hline $\mathbf{C}$ & $\mathbf{M n}$ & $\mathbf{S i}$ & $\mathbf{P}$ & $\mathbf{S}$ & $\mathbf{C r}$ & $\mathbf{N i}$ & Mo \\
\hline 0.20 & 1.57 & 1.65 & 0.015 & $<0.001$ & $<0.001$ & $<0.001$ & $<0.001$ \\
\hline $\mathbf{C u}$ & $\mathrm{Ti}$ & $\mathbf{C o}$ & $\mathbf{B}$ & $\mathbf{P b}$ & $\mathbf{V}$ & $\mathbf{W}$ & $\mathbf{A l}$ \\
\hline 0.007 & 0.002 & 0.003 & $<0.001$ & $<0.001$ & 0.001 & $<0.001$ & 0.058 \\
\hline
\end{tabular}
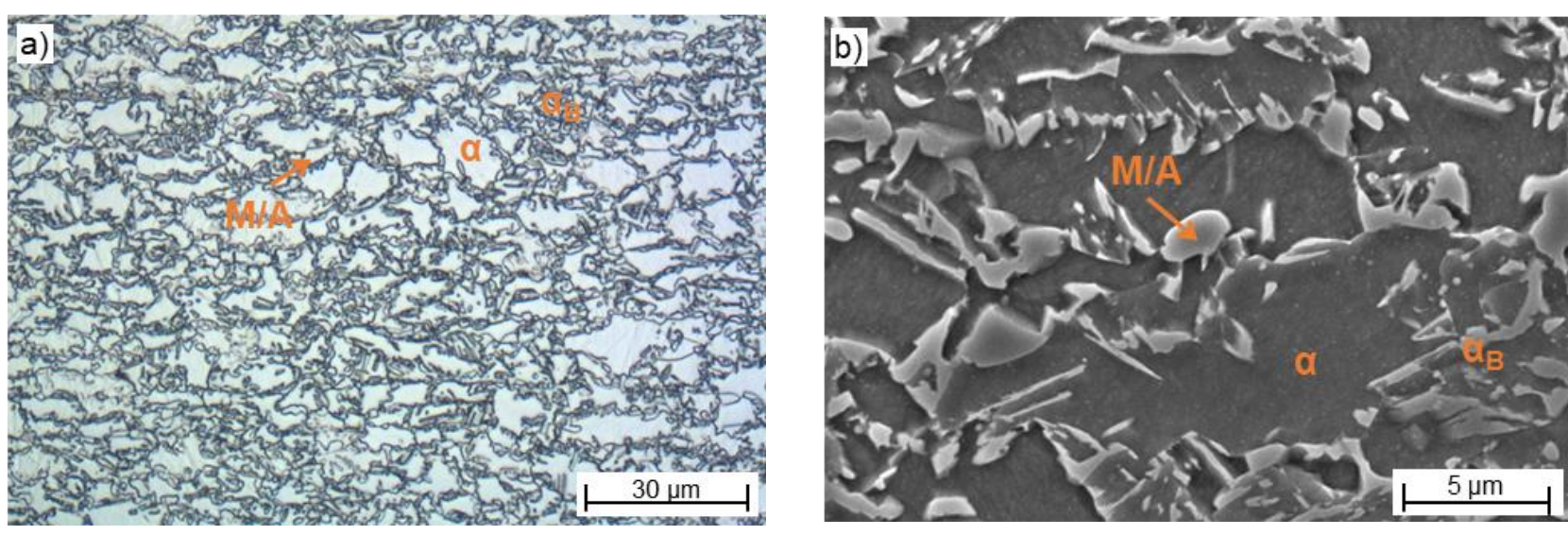

Figure 1 The microstructure of steel TRIP 780 as delivered, longitudinal direction, etched in Nital,

a) LM, b) SEM-SE.

Metallographic sections of the samples were evaluated after polishing and etching in $2 \%$ Nital. Metallographic analysis was performed using an Olympus IX70 light metallographic microscope (LM) and using a JEOL $6490 \mathrm{LV}$ scanning electron microscope (SEM) in mode of secondary electron (SE). The microstructure consists of ferrite $(\alpha)$, bainite $\left(\alpha_{B}\right)$ and retained austenite $(R A)$, resp. M/A constituent (Figure 1). The photo documentation of microstructure by SEM makes possible to observe the microstructure at higher magnification 
and higher resolution compared to LM, but even SEM does not make it possible to obtain complete information on the occurrence of martensite or retained austenite in structural components referred to as the M/A constituent. The material showed high metallurgical purity. In the microstructure, there were complex inclusions based on alumina and calcium sulphide, inclusions based on aluminium arranged in the rolling direction and sharp-edged inclusions of titanium nitride. The size of individual inclusions was around $5 \mu \mathrm{m}$, the size of inclusions in rows reached up to $35 \mu \mathrm{m}$. The proportion of retained austenite (RA) in TRIP steel was determined by X-ray diffraction analysis. The proportion of retained austenite was found to be $14.3 \mathrm{vol}$. \%.

\subsection{Mechanical properties}

The samples were cut from $1.17 \mathrm{~mm}$ thick metal sheet in the longitudinal direction by electro spark cutting and surface was ground on a magnetic grinder. A sample without deformation or the influence of hydrogen was used as the initial state. The original parameters of the samples were: width $b=10 \mathrm{~mm}$, thickness $a=1.17 \mathrm{~mm}$ and area $S_{0}=11.7 \mathrm{~mm}^{2}$. The initial measured lengths $L_{0}$ of the original samples were $50 \mathrm{~mm}$.

Subsequently, a part of the samples was pre-deformed by $5 \%$ of the total deformation, i.e. extended by $2.75 \mathrm{~mm}$, and a part of the samples was pre-deformed by $10 \%$ of the total deformation, i.e. extended by $5.5 \mathrm{~mm}$, for induction a partial transformation of retained austenite to martensite. The initial measured lengths $L_{0}$ of the pre-deformed samples were $55 \mathrm{~mm}$. The pre-deformation was performed at a loading rate of $2 \mathrm{~mm} \cdot \mathrm{min}^{-1}$, but the fracture test itself took place at a loading rate of $0.1 \mathrm{~mm} \cdot \mathrm{min}^{-1}$, which corresponds to a deformation rate of $10^{-5} \mathrm{~s}^{-1}$. Tensile diagrams of these samples are plotted in Figure 2. Mechanical properties of samples without hydrogen as yield strength (YS), tensile strength (TS) and the elongation at fracture (A) are given in Table 2. It is clear from the data that the yield strength significantly increases with deformation; by $34 \%$ after $5 \%$ of deformations and $60 \%$ after $10 \%$ of deformations compared to the initial state (IS). The tensile strength increases slightly by $2 \%$ after $5 \%$ of deformations and $6 \%$ after $10 \%$ of deformations. On the contrary, the elongation decreases, by $14 \%$ after $5 \%$ of deformations and $32 \%$ after $10 \%$ of deformations.

In the case of TRIP steels retained austenite transforms to martensite during deformation. It was necessary to find out how this transformation will affect the mechanical properties of TRIP steel during hydrogenation. A loading rate of $0.1 \mathrm{~mm} \cdot \mathrm{min}^{-1}$ is important to ensure sufficient time for hydrogen to be transferred to the hydrogen traps. A special cell was used for tensile testing of samples during simultaneous hydrogenation and deformation. A platinum-plated tungsten mesh was placed around the tensile sample, which served as an anode and was connected to a voltage source. A sample was attached as a cathode. The electrolyte was poured after closing the cell. This electrolyte was prepared as a $0.05 \mathrm{M}$ solution of sulfuric acid $\left(\mathrm{H}_{2} \mathrm{SO}_{4}\right)$ with $1 \mathrm{~g}$ of potassium thiocyanate (KSCN) in one liter of distilled water. The prepared samples were mounted in the tensile test machine and hydrogenation started. The electrolytic hydrogenation took place at a current density of $1 \mathrm{~mA} \cdot \mathrm{cm}^{-2}$, a total current of $11 \mathrm{~mA}$ and a voltage of $28.9 \mathrm{~V}$. The resulting mechanical properties were very low and the tensile diagrams differed significantly from previous tests, which were performed on undeformed samples in the work [18]. Subsequently, further tests were performed for both 5\% and $10 \%$ pre-deformation on samples pre-charged by hydrogen. In these tests, the samples were first pre-hydrogenated for 4 hours in the same solution and the same current density as in the simultaneous hydrogenation and then loaded at a loading rate of $0.1 \mathrm{~mm} \cdot \mathrm{min}^{-1}$. Figure 3 shows the tensile diagrams of a sample that was pre-deformed (5\%) and simultaneously hydrogenated, and a sample that was pre-deformed $(5 \%)$, subsequently prehydrogenated and then loaded to fracture. After evaluating the mechanical properties, the hydrogen embrittlement index IE in \% was calculated by equation 1.

$I E=\frac{A_{0}-A_{H}}{A_{0}} \cdot 100 \%$

The hydrogen embrittlement index refers to the change in elongation at fracture between non-hydrogenated $A_{0}$ and hydrogenated test specimens $A_{H}$ relative to the elongation at fracture of the initial state without hydrogen $A_{0}$. The values of mechanical properties and hydrogen embrittlement index values for samples after $5 \%$ and $10 \%$ pre-deformations are plotted in Table 2. 
It can be deduced from the tensile diagrams (Figure 3) that during the simultaneous hydrogenation and deformation it was not possible to determine the yield strength YS. Comparing the samples after $5 \%$ predeformation without hydrogen and after pre-hydrogenation show that the YS increased by $41.2 \mathrm{MPa}$, the tensile strength TS decreased by $104.9 \mathrm{MPa}$ and the elongation at fracture At decreased significantly by $23.2 \%$. The hydrogen embrittlement index was equal to $76.2 \%$ for simultaneous hydrogenation and to $78.1 \%$ for pre-hydrogenation. Similar to $5 \%$ pre-deformation, the analysis was also performed for $10 \%$ predeformation, namely: the yield strength decreased by $28.8 \mathrm{MPa}$, the tensile strength decreased by $68.0 \mathrm{MPa}$ and the elongation at fracture At decreased by $22.2 \%$. The hydrogen embrittlement index was equal to $86,2 \%$ for pre-hydrogenation. The values of mechanical properties found during their simultaneous hydrogenation are for information only.

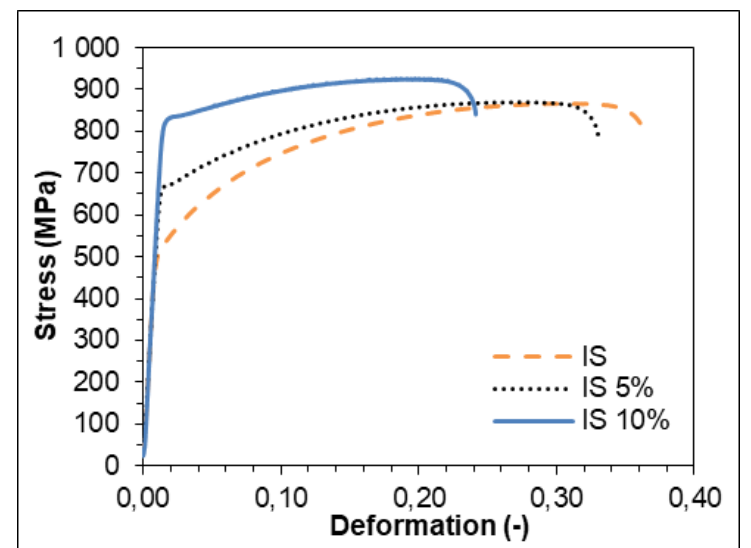

Figure 2 Tensile diagrams of samples in initial state (IS), in initial state after $5 \%$ pre-deformation (IS 5 $\%$ ), in initial state after $10 \%$ pre-deformations (IS $10 \%$ ) without hydrogen

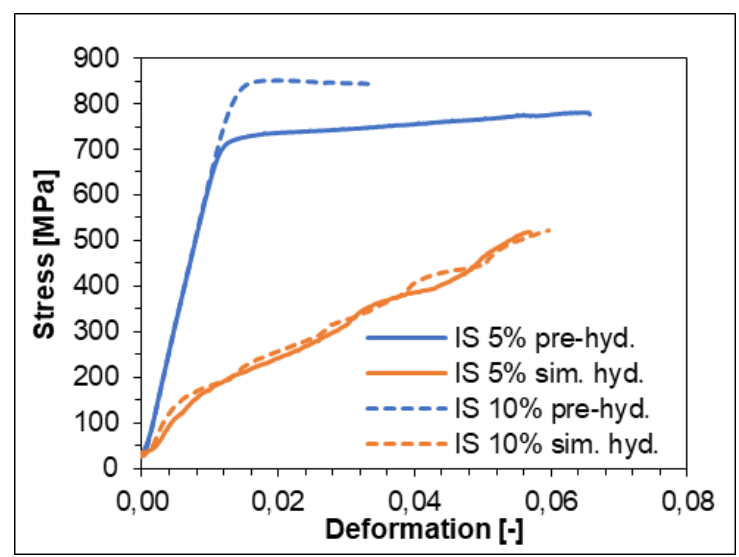

Figure 3 Tensile diagrams of samples after simultaneous hydrogenation and after prehydrogenation and deformation

Table 2 Mechanical properties of samples in initial state and after pre-deformation and hydrogenation

\begin{tabular}{|l|c|c|c|c|}
\hline Sample/state & $\begin{array}{c}\text { YS } \\
\text { (MPa) }\end{array}$ & $\begin{array}{c}\text { TS } \\
\text { (MPa) }\end{array}$ & $\begin{array}{c}\text { At } \\
(\%)\end{array}$ & $\begin{array}{c}\text { IE } \\
(\%)\end{array}$ \\
\hline initial state (IS) & 504 & 868 & 34.5 & - \\
\hline IS after 5\% pre-deformation (IS 5\%) & 674 & 884 & 29.7 & - \\
\hline IS 5\% simultaneous hydrogenation & - & 538 & 7.1 & 76.2 \\
\hline IS 5 \% after pre-hydrogenation & 716 & 780 & 6.5 & 78.1 \\
\hline IS after 10\% pre-deformation (IS 10\%) & 806 & 918 & 24.2 & - \\
\hline IS 10\% simultaneous hydrogenation & - & 538 & 7.9 & 67.4 \\
\hline IS 10\% after pre-hydrogenation & 834 & 850 & 3.4 & 86.2 \\
\hline
\end{tabular}

Note: Elongation at fracture At is the ratio of the total elongation of the sample at fracture of the sample and the length $L_{0}$ expressed in \%.

According to [5], for samples that were not pre-deformed and were co-hydrogenated and loaded at a current density of $1 \mathrm{~mA} \cdot \mathrm{cm}^{-2}$ in the same type of solution, the value of the hydrogen embrittlement index was $90.0 \%$. Hydrogen embrittlement index values were expected to be higher. In the case, when $5 \%$ pre-deformation was performed on the samples and the samples were pre-hydrogenated, the hydrogen embrittlement value was $83.3 \%$. Compared to this work, the values in Table 2 are comparable. Comparing the values without hydrogen and after hydrogenation for $5 \%$ and $10 \%$ pre-deformation, it is clear that hydrogen in this case increased the yield strength, decreased the tensile strength and significantly reduced the elongation at fracture. From the 
point of view of hydrogen embrittlement, it is clear that hydrogen causes greater embrittlement at a higher degree of deformation, and thus at a higher proportion of martensite in the microstructure.

\subsection{Fracture analysis}

Fractographic analysis was performed on a JEOL 6490LV scanning electron microscope in secondary electron mode. The mixture of transgranular cleavage and ductile fracture in the middle and the dimple morphology, i.e. the transgranular ductile fracture, along the edges formed the fracture surface of the samples in the initial state without hydrogen. The character of the fracture surfaces of the samples after $5 \%$ and $10 \%$ predeformation was similar to the initial state without hydrogen.

Figure 4 shows the fracture surface of samples that were $5 \%$ pre-deformed and then simultaneously hydrogenated and loaded to fracture. In detail A it can be seen there is mainly a quasi-cleavage fracture on the edge, which is formed by finer facets compared to the common cleavage fracture and is typical for hydrogen embrittlement. In further details transgranular mixed fracture is visible (detail B), but quasi-cleavage fracture is predominant in the centre bands of the sample (detail C). Fisheyes (detail D), which are characteristic for hydrogen embrittled steels, occurred on fracture surface almost all hydrogenated samples. Fisheyes are formed in the presence of larger inclusions, around which a quasi-cleavage fracture spreads and which passes mainly into a transgranular ductile fracture. The samples, which were $5 \%$ pre-deformed, pre-hydrogenated and then loaded, showed a very similar character of fracture surfaces. In the samples pre-hydrogenated with $10 \%$ pre-deformation, a part of the fracture surface was formed by a purely transgranular cleavage fracture.

\section{CONCLUSIONS}

Hydrogen in deformed TRIP steel has changed many of steel properties. It can be stated that hydrogen increased the yield strength, but on the contrary reduced the tensile strength and significantly reduced the elongation at fracture. It is clear from the results of the hydrogen embrittlement index that hydrogen acted worse on samples that were subjected to higher deformation. The occurrence of deformation-induced martensite in the microstructure of TRIP steels contributed significantly to the susceptibility of these steels to hydrogen embrittlement. Fractographic analysis showed typical features caused by hydrogen, such as fisheyes. The hydrogenated samples showed a continuous area of quasi-cleavage fracture at the edges. Quasi-cleavage fracture occurred also in the middle of the cross-section in row microstructure. Based on this work, it can be stated that TRIP 780 steels are highly susceptible to hydrogen embrittlement, which significantly impairs their mechanical properties.
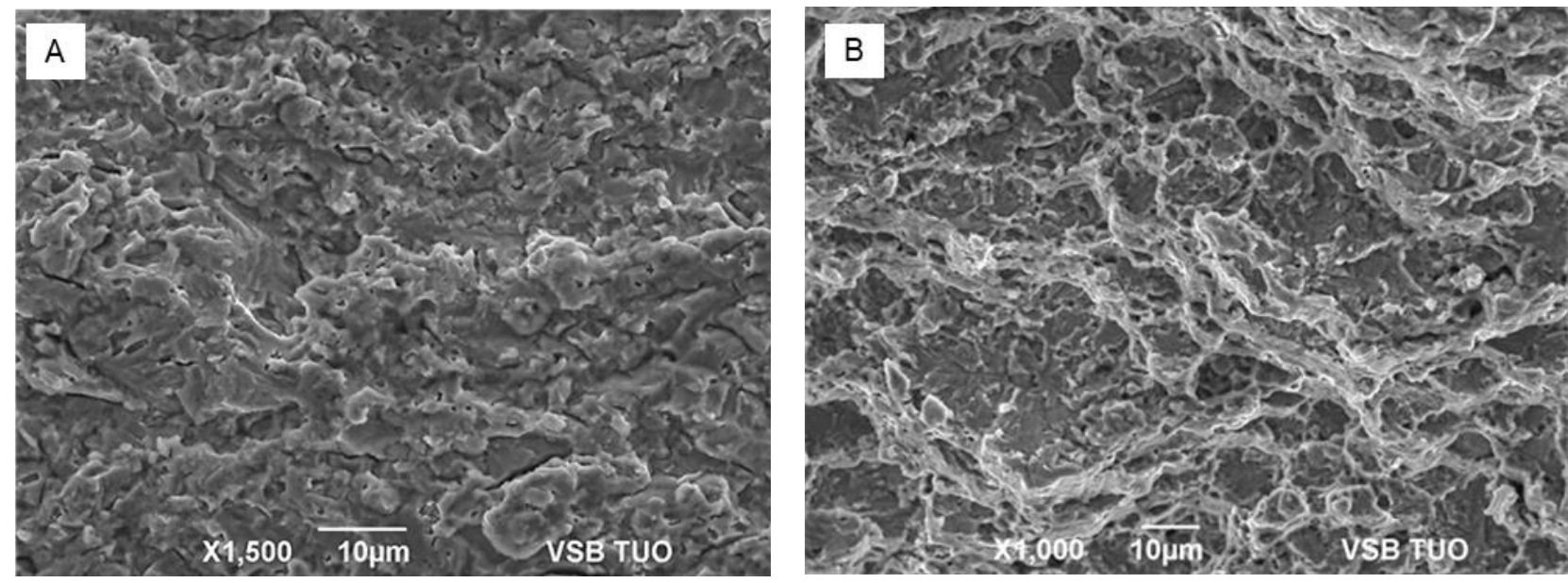

Figure 4/1 Fracture area of samples after $5 \%$ pre-deformation, simultaneous hydrogenation and deformation 

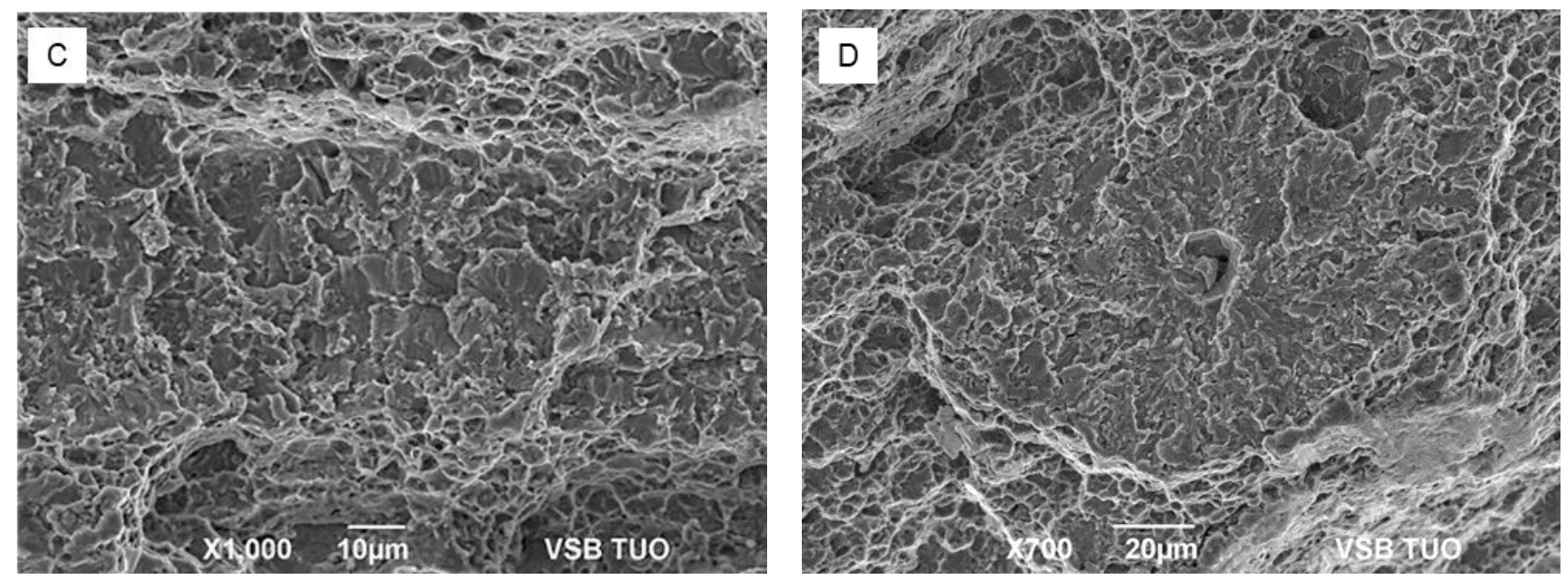

Figure 4/2 Fracture area of samples after $5 \%$ pre-deformation, simultaneous hydrogenation and deformation

\section{ACKNOWLEDGEMENTS}

This paper was created with the contribution of the projects Student Grant Competition "SP2020/58 Development of methods of structural analysis, testing of mechanical properties and non-destructive testing of advanced materials" and "SP2020/39 Specific research in the metallurgical, materials and process engineering".

\section{REFERENCES}

[1] DE COOMAN, B. C. Structure-properties relationship in TRIP steels containing carbide-free bainite. Current Opinion in Solid State and Materials Science. 2004, vol. 8, no. 3-4, pp. 285-303.

[2] GALÁN, J., et al. Advanced high strength steels for automotive industry. Revista de metalurgia. 2012, vol. 48, no. 2, pp. 118-131.

[3] LYNCH, S. P. Hydrogen embrittlement (HE) phenomena and mechanisms. In: Interrante, C. G., ed. Stress Corrosion Cracking. Cambridge: Woodhead Publishing, 2011. pp. 90-130.

[4] LI, X., et al. Effect of pre-strain on hydrogen embrittlement of high strength steels. Materials Science and Engineering A. 2014, vol. 616, pp. 116-122.

[5] VÁŇOVÁ, P., et al. The effect of hydrogen and deformation on the mechanical properties of TRIP 780 steel. In: METAL 2018 - 27th International Conference on Metallurgy and Materials, Conference Proceedings. Ostrava: TANGER, 2018, pp. 897-902. 